\title{
Hybrid Visualization for Deep Insight into Knowledge Retention in Firms
}

\author{
Lourdes Sáiz ${ }^{1}$, Miguel A. Manzanedo ${ }^{1}$, Arturo Pérez $^{2}$, Álvaro Herrero $^{1}$ and Emilio \\ Corchado $^{3}$ \\ ${ }^{1}$ Department of Civil Engineering, University of Burgos \\ C/ Francisco de Vitoria s/n, 09006 Burgos, Spain \\ ${ }^{2}$ Investigador Programa Torres Quevedo, TTT Diseño, Comunicación y Contenidos, S.L. \\ Reyes Católicos, 41, 1, 09005 Burgos, Spain \\ ${ }^{3}$ Departamento de Informática y Automática, Universidad de Salamanca \\ Plaza de la Merced, s/n, 37008 Salamanca, Spain \\ \{1saiz,mmanz,ahcosio\}@ubu.es, arrturo@hotmail.com, escorchado@usal.es
}

\begin{abstract}
Neural projection models are applied in this study to the analysis of Human Resources (HR) from a Knowledge Management (KM) standpoint. More precisely, data projections are combined with the glyph metaphor to analyse KM data and to gain deeper insight into patterns of knowledge retention. Following a preliminary study, the retention of specialized employees in hi-tech companies is investigated, by applying the configurational approach of Strategic HR Management. The combination of these two aforementioned techniques generates meaningful conclusions and the proposal is validated by means of an empirical study on a real case study related to the Spanish hi-tech sector.
\end{abstract}

Keywords: Unsupervised Neural Networks, Glyph Metaphor, Knowledge Management, Knowledge Retention, Human Resources Management.

\section{Introduction}

Knowledge Management (KM) [1] means that organizations can capture and share the collective experience and the know-how (knowledge) of their employees and apply their knowledge in intelligent ways [2]. However, before an organization can successfully apply a KM methodology, it first has to develop and to implement its knowledge infrastructure [3]. These knowledge infrastructures consist of three central dimensions: people, organizational and technological systems.

It is no exaggeration to say that in an environment such as today's, where everything changes at great speed and almost nothing remains static, knowledge emerges as the key factor in any economy [4]. Knowledge not only means that we can advance, but that we can adapt to the unceasing change that happens around us, which 
are bound to increase in the future. Equally, the firm inevitably requires "specific/singular" knowledge, which will permit it to pursue excellence alongside others. This class of first-level knowledge is held by a small number of people, without forgetting that more select and sophisticated knowledge is required, if possible, in pioneering firms, such as those analyzed in this study, in an area where the offer is, unfortunately, further and further away from satisfying demand.

Here, the full force of the need is felt, firstly, to recruit those employees with specialist knowledge and, secondly and most importantly, to retain them and to capitalize on their potential. As the visionary noted [5], it is more difficult to lose human capital, when the firm develops its own specific abilities that are difficult to transfer. The negative consequences of that fact are accentuated, when key employees, leaders or those with a high performance are affected, because it is very difficult to replace workers with a high contribution without affecting global performance [6] [7].

In an effort to avoid these situations, the different configurations that motivate employees to remain in a firm are listed for review. Beforehand, it should be mentioned that the term configuration is linked to the configurational approach of Strategic Human Resource Management [8], according to which there is a coherent set of HR practices with strategic objectives that contribute to the competitiveness and the survival of the firm. Thus, the adjustment between employee and organization is quite a good predictor of the intention to remain committed to the firm or to leave it [9], such that high levels of adjustment are more likely to lead to the subsequent retention of employees. Both, competitive salaries as well as systems of financial rewards, are the most significant factors in the decision to leave the organization or to seek new opportunities [10], [11].

High salaries can act as a signal to retain qualified employees, protecting the firm from losing its investments in training, and avoiding extra expenditure on vacancies caused by departures [12]. Furthermore, high levels of retention are achieved through the offer of a salary, linked to other investments, such as the creation of structures to help new workers to acclimatize to their jobs and their environment, efforts to reduce unequal treatment between workers, and a commitment to small but incremental opportunities for development and progress [13]. [14] showed that the announcement of plans for share options by organizations slowed voluntary departures.

Moreover, certain environments are more favorable for employee retention than others [15]. Thus, young workers demand flexible and informal working environments [16]. Employees may be less willing to leave an organization with a system of social practices that reinforces company identity. The creation of a strong social atmosphere helps to encourage companionship between employees, leading to low rates of abandonment, as they feel reluctant to leave their friends [17], because a sense of belonging is reinforced [18] and, equally, because a high social implication in the organization is positively related to commitment [19] [20] [21] [22].

It appears clear that the more satisfied and the more motivated an individual feels, the more difficult it will be for that individual to leave the organization; the concept of commitment could be extended as far as the degree to which employees see the organization as a source of satisfaction [23]. Relations with companions are, generally, linked to on-the-job satisfaction, which is in turn related to a sense of belonging to the firm [24] [25]. A culture that emphasizes interpersonal relations will 
attract professionals, more than another that is characterized by the value of its tasks [26]. The experiment conducted by [27], with a group of key employees, showed how they really consider themselves as relevant members when they belonged to a working environment with a high degree of interaction between people; when the work gave them autonomy, challenges, feedback, opportunities for development and the possibility to demonstrate their skills; and, also, if there was provision for continuous training and education.

Therefore, the firm can significantly increase its employee retention rate, deepening both satisfaction and motivation [28] [29]; at the same time as reducing the costs of employee departures [30] [31], as it has been demonstrated that both labor and extra-labor aspects influence the decision to remain in the firm [32]. In consequence, the configuration of employee retention, with what has been shown above, includes the components associated with employee adjustment to the organization, above-average remuneration in relation to competitors, harmonization with organizational culture, investments and support for recent arrivals, and social training events and actions.

Based on the ideas discussed above, the authors conducted a broad analysis of factors [33], ranging across advanced HR practices to explanations of firm performance. In doing so, our study [33] focused on intermediate indicators, such as employee characteristics, organizational capabilities and some other internal features. The factors in the study consisted of five settings for HR practices (acquisition, development, commitment, retention and flexibility), five employee features (human, social capital, organizational capital, motivation and turn over), four organizational capabilities (knowledge creation, knowledge application, organizational flexibility and information technologies), and some other internal features (strategic vision, HR emphasis, heterogeneity, and task-associated technology). Only those decisions relating to the acquisition of specialized personnel and employee-retention rates and the configuration of those two points (Strategic HR Management [34]) were analyzed.

Going one step further, the present research thoroughly analyzed those features related to Knowledge Retention policies. To do so, neural projection models [1] [35] [36], described in section 2, were trained and applied to generate intuitive visualizations of the data by reducing dimensionality. The instances are depicted in different colors and symbols to help extract conclusions, incorporating further meaningful information into those projections. Further details about the experiments are provided in section 3. Section 4 presents the obtained results while conclusions and future work are outlined in section 5.

\section{Neural Projection Model}

Projection models [37] operate on the spatial coordinates of high-dimensional data, in order to project them onto lower dimensional spaces. The main goal is to identify the patterns that exist across dimensional boundaries by identifying "interesting" directions, in terms of any specific index or projection. Such indexes or projections are, for example, based on the identification of directions that account for the largest variance of a data set -i.e. Principal Component Analysis (PCA) [38], [39]- or the 
identification of higher-order statistics such as the skew or kurtosis index -i.e. Exploratory Projection Pursuit (EPP) [37]. Having identified the most interesting projections, the data is then projected onto a lower dimensional subspace, plotted onto two or three dimensions, which makes it possible to examine its structure with the naked eye.

The combination of projection techniques together with the use of scatter plot matrices is a very useful visualization tool to investigate the intrinsic structure of multidimensional data sets, allowing experts to study the relations between different components, factors or projections, depending on the technique that is applied.

The solution proposed in this research applies an unsupervised neural model called Cooperative Maximum Likelihood Hebbian Learning (CMLHL) [2] [35]. It is based on Maximum Likelihood Hebbian Learning (MLHL) [2] [35], and introduces the application of lateral connections [2] [35] derived from the Rectified Gaussian Distribution [40]. This connectionist model has been chosen because it reduces the data dimensionality while preserving the topology in the original data set. Considering an $\mathrm{N}$-dimensional input vector $(x)$, and an M-dimensional output vector ( $y$ ), with $W_{i j}$ being the weight (linking input $j$ to output $i$ ), then CMLHL can be expressed as:

1. Feed-forward step:

$$
y_{i}=\sum_{j=1}^{\mathbf{N}} W_{i j} x_{j}, \forall i
$$

2. Lateral activation passing:

$$
y_{i}(t+1)=\left[y_{i}(t)+\tau(b-A y)\right]^{+} .
$$

3. Feedback step:

$$
e_{j}=x_{j}-\sum_{i=1}^{M} W_{i j} y_{i}, \forall j
$$

4. Weight change:

$$
\Delta W_{i j}=\eta \cdot y_{i} \cdot \operatorname{sign}\left(e_{j}\right)\left|e_{j}\right|^{p-1} .
$$

Where: $\eta$ is the learning rate, $\tau$ is the "strength" of the lateral connections, $b$ the bias parameter, $p$ a parameter related to the energy function and $A$ a symmetric matrix used to modify the response to the data [2] [35]. The effect of this matrix is based on the relation between the distances separating the output neurons. This neural projection model has been previously applied to the KM field [1].

\section{Experimental Study}

In its empirical validation of acquisition and retention factors, this study looked at 126 high-tech organizations in Spain. $267 \mathrm{R} \& \mathrm{D}$ employees from these firms were 
surveyed, in order to analyze their HR strategies and subsequently improve the status of the analyzed firms.

The typical profile of these hi-tech firms would be an organization with 266 employees, manufacturing products and services (111 from the 126 organizations under study). A total of $47 \%$ of firms claimed that they innovated in both products and services, running 124 annual R\&D programs. A total of $44 \%$ of the firms were members of a corporate group, $16 \%$ of which were international.

As only the HR retention factors are considered in the present study, features relating to these factors are analyzed in the surveyed data and described in Table 1.

Table 1. Analyzed components for retention factor.

\section{Retention factor}

$\mathrm{C} 1$ : Candidates are selected according to their fitting with the firm.

$\mathrm{C} 2$ : Employees match the organizational culture.

C3: New employees are supported.

C4: Social and outdoor activities are sponsored by the firm for employees to know each other.

C5: Higher salaries than competitors are offered to retain employees.

Five components were used to define the retention factor. All these features have discrete values that range from 1 (strongly low) to 5 (strongly high). As a result, five features from each of the (126) firms were collected for the dataset.

Two analyses were done for the purpose of an interesting comparison. In the first one, each component or retention criteria was weighted with an identical value $(0.20)$, which established a typology or categorization of firms in the category as: Excellent, High, Normal, Low and Poor. An "excellent" firm in the retention configuration means that all components obtained a score of between 4.5 and 5. The firms that fell into the "high" category scored between 3.5 and 4.4. in all criteria; while the "normal" consideration was for companies with values of between 2.8 and 3.4; the "low" category for scores between 2 to 2.7 and the "poor" category, whenever the score was below 2 .

The second analysis consisted in assigning a greater weight to one of the components (0.4), leaving the rest equal (0.15), and so on, respectively, for each of the criteria. The aim is, in this way, to evaluate the overall consequences and effects that each component has on the knowledge retention factor, visualizing the variations that are shown. The results of this comparative study help us determine the specific measures that the firm should take, in areas such as employee selection, actions to encourage employees to identify with the organizational culture, protocols and measures for the integration of new employees and components to determine attractive salaries. All of these to achieve efficiency in the configuration of HR retention in the firm. 


\section{Results \& Analysis}

This section comprises an analysis of the best projections obtained in the abovedescribed experimental study by applying the CMLHL model to the data on the knowledge retention factor. Fig. 1 shows the groups with their labels $(1.1,1.2 \ldots)$, projected by means of CMLHL and according to the retention factor.

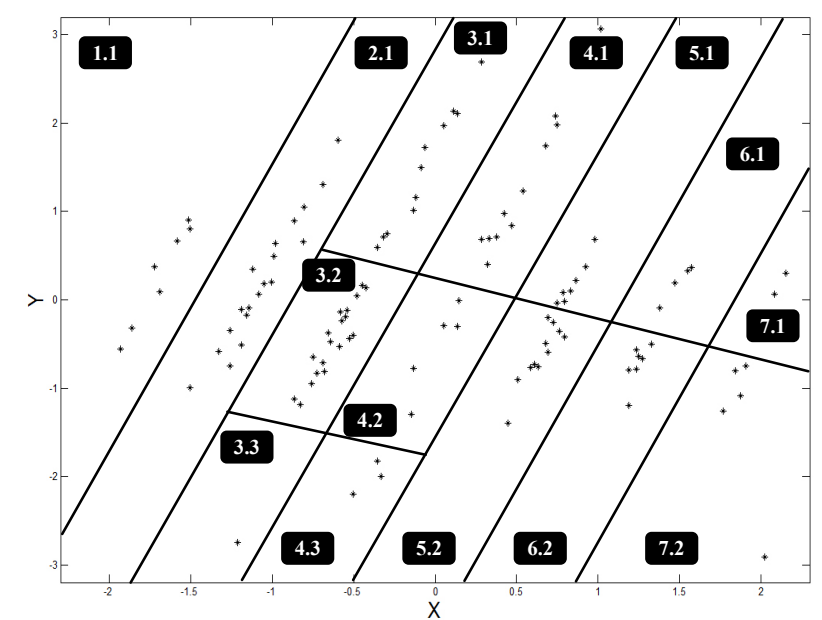

Fig. 1. CMLHL projection of the Retention Factor dataset.

\subsection{Analysis 1}

Firstly, the combination of CMLHL and glyph depiction was applied to the equallyweighted features of knowledge retention. With regard to the first study, the application of the tool to the data and its subsequent interpretation immediately identified the different situations that can arise in the configuration of retention. In consequence, it provides information to take well-judged decisions for employee retention in firms with similar characteristics to those in this study. It is a preliminary step of transcendental importance to achieve the business objectives of competitiveness and survival.

Analyzing the five components all together (Fig. 2), the firms that are found to the right of the diagram present excellent knowledge retention, a position that starts to worsen as we move towards the left, such that those situated in the center represent high retention, which becomes normal as we move in that same direction, until we arrive at the low and poor categories in the positions furthest to the left. Simultaneously, the tool also shows the excellent firms situated in the lower part of the diagram, the high firms in the intermediate zone, and the remainder in the upper zone. 


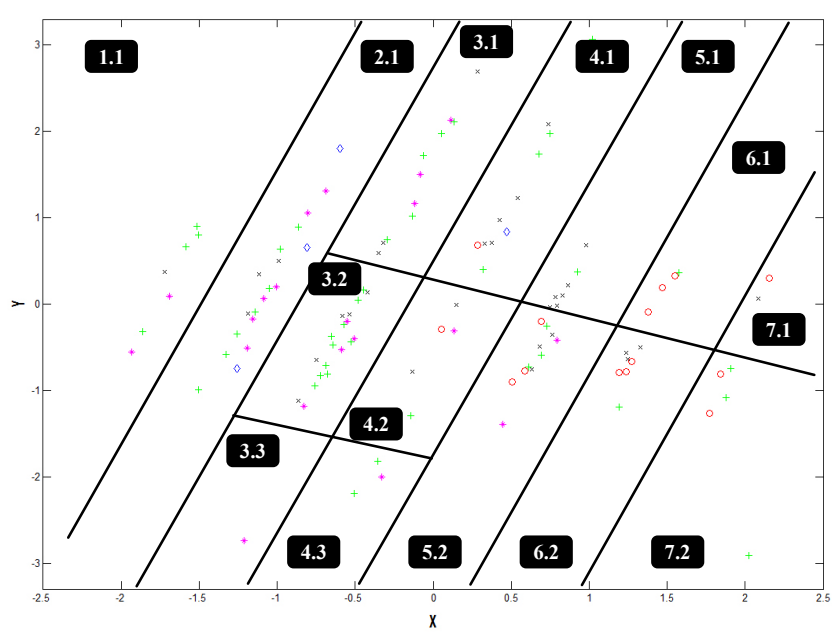

Fig. 2. CMLHL projection of the Retention Factor dataset for equally-weighted features.

The grid generated by the application of the tool is very useful to classify firms and to place them with respect to the configuration of knowledge retention and, in consequence, to adopt the decisions that contribute to maintaining and to correcting these locations. In other words, an acceptable interpretation of the relevant data will highlight the existing configuration and will contribute sufficient knowledge, if necessary, in order to change position, determining not only the components that obtain little value, but also stressing the measures to be able to change undesirable locations, or in other words, firms in weaker categories. These measures, in R\&D companies are connected with the selection of employees in accordance with the global adjustment of the firm, its clear identification with organizational culture, integration, the development of activities for recently contracted employees and a higher salary offer than its competitors.

\subsection{Analysis 2}

Fig. 3 shows the CMLHL projection of the analysis of firms, according to the retention factor, varying the component weights. The overvalued factor is marked with a plus character ("+"). That is, "+C1" means that the component 1 (See Table 1) was given a higher weight than others.

In addition to the interpretation of the positions that the firms occupy in the projection (Fig. 3), the study was complemented by a second analysis, with the objective of comparing the variation or effect of each component on the overall retention factor. To do so, each component of the retention configuration, starting with the first one and finishing with the fifth one, were iteratively weighted with a higher value $(0.50)$ with regard to the others $(0.15$ for each of the four remaining ones) and its effect on the initial typology was studied (where all the criteria were 
weighted with an identical value). In this way, five different experiments were conducted, varying the weight from the first to the fifth component.

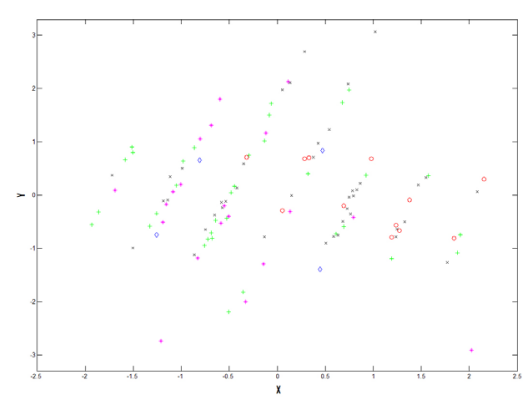

Fig. 3.1 CMLHL projections of the retention factor dataset: overvalued component 1 $(+\mathrm{C} 1)$.

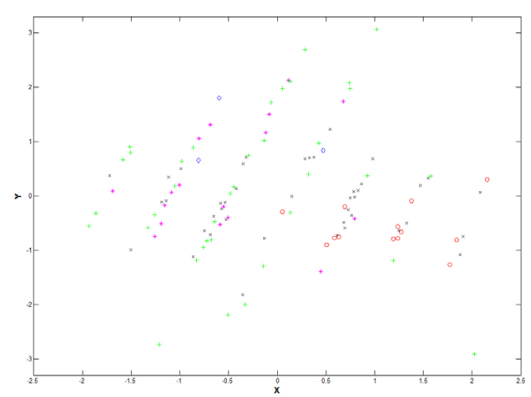

Fig. 3.3 CMLHL projections of the retention factor dataset, overweighting component 3 $(+\mathrm{C} 3)$.

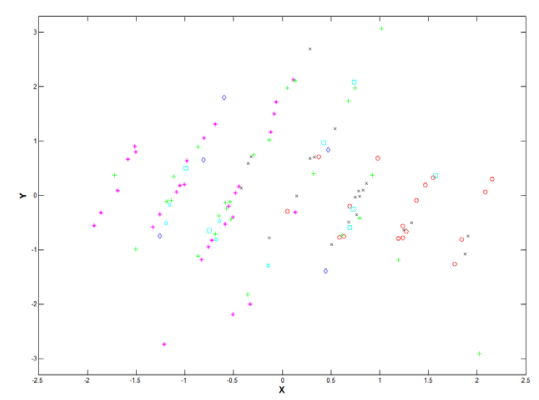

Fig. 3.5 CMLHL projections of the retention factor dataset, overweighting component 5 $(+\mathrm{C} 5)$

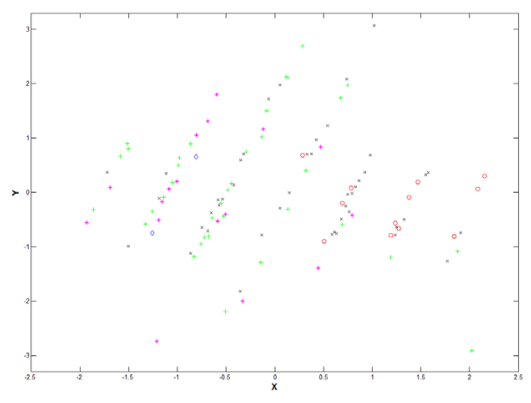

Fig. 3.2 CMLHL projections of the retention factor dataset, overweighting component 2 $(+\mathrm{C} 2)$.

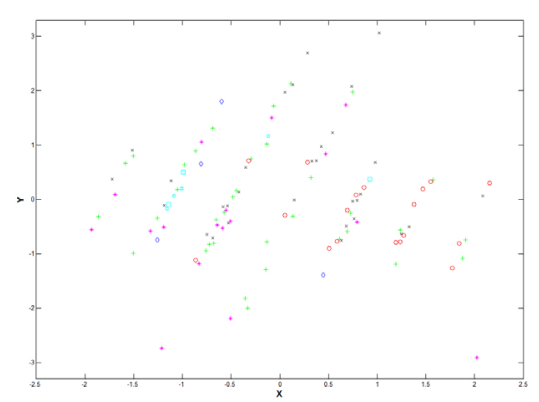

Fig. 3.4 CMLHL projections of the retention factor dataset, overweighting component 4. $(+\mathrm{C} 4)$.

The results were very revealing. Starting with Fig. 2, fourteen clearly identified groups or sections were observed, each of which represents, according to this study, 
two revealing aspects of the firms in the sample, their category or the level that they reach in the retention configuration, as well as the high values that correspond with excellent or very good human resource retention, while the very low values represent the contrary situation, and their localization.

When the highest weighting is attached to component $\mathrm{C} 1$ (selection of individuals according to the global adjustment with the firm), the results present the following changes (Fig. $3.2+\mathrm{C} 1$ ): in group 1.1, one firm moves from a low to a normal category; in 2.1, one firm changes from poor to low and two others change from normal to high categories; in group 3.1, one firm changes from low to normal, two from normal to high and one from high to excellent; in 3.2, two firms move from normal to high; in section 4.1, one firm changes from normal to high and one more from high to excellent; in 4.2, one moves from normal to high; in group 5.1, one passes from high to excellent; in group 5.2, another changes from normal to high; in group 6.2 as well as in group 7.2, a single firm changes from high to excellent. There are a total of sixteen changes, in all of which the firms improve the retention configuration with regard to their initial situation.

In the case of the greater weighting given to $\mathrm{C} 2$ (employees identify with the organizational culture), the variations are (Fig. $3.2+\mathrm{C} 2$ ) as follows: in group 2.1, one firm moves from poor to low and another from normal to high; in 3.1, three firms vary from low to normal, while another moves from normal to high; in 3.2, one firm changes from low to normal, while another three move from normal to high; in group 4.1, one firm changes from poor to low and another from normal to high; in 4.2, one moves from low to normal, another from normal to high, and yet another from high to excellent; in group 5.1, one company switches the category of normal for high, and another switches from high to excellent; in group 5.2, two firms change from normal to high; in group 6.1, one company moves from normal to high; in 6.2, another moves from high to excellent, in 7.1, one firm changes from high to excellent and in group 7.2 , one firm passes from normal to high. The changes affect twenty-three firms, all of which improve the retention configuration, as in component $\mathrm{Cl}$.

C3 (the firm provides support for the incorporation of recently contracted employees) is given a greater weighting in retention, but with the following changes (Fig. $3.2+\mathrm{C} 3$ ): in group 1.1, one single firm moves from low to normal; in 2.1, one firm changes from poor to low, and two others from normal to high; in group 3.2, one firm changes from low to normal and four more from the normal to the high category; group 4.2 presents a change from low to normal; in 4.3, one changes from poor to normal and another from normal to high; in group 5.2, three companies change from normal to high and another from high to excellent; finally, in group 6.2, one firm changes from high to excellent. Here too, the changes are improvements for all sixteen firms.

With regard to attributing greater weight to component $\mathrm{C} 4$ (promotion of social events and activities so that workers relate and get to know each other), the results observed are as follows (Fig. $3.2+\mathrm{C} 4$ ):

In group 1.1, four firms change from normal to low and one other from high to normal; in 2.1, another four firms move from normal to low and two more from high to normal; in 3.1, one company changes from normal to high, in 3.2 , six firms change from normal to low and three more from high to low; in group 4.1, two companies switch from the high to the normal category and another one from excellent to high; 
10 Lourdes Sáiz, Miguel Á. Manzanedo, Arturo Pérez, Álvaro Herrero and Emilio

Corchado

in 4.2, one moves from normal to low, and the same happens in 4.3; in 5.2, one firm changes from low to poor and another from excellent to high. In view of these data, criterion $\mathrm{C} 4$ is clearly the only one that provokes more unfavorable or retrograde situations, affecting 38 companies, at the same time as it is shown to be the component that provokes the most changes in the firms and is the one that differentiates or discriminates between the data under analysis more than any other. This is because the firms under study clearly present inferior values under this criterion to those of the other components, except in groups 6.1, 6.2, 7.1 and 7.2 that reach the highest possible value.

Finally, when C5 (offers higher salaries than competitors) is given a greater weighting, the following transformations appear (Fig. $3.2+\mathrm{C} 5$ ): in 1.1, one firm moves from normal to high; in 2.1., another firm varies from low to normal; in 3.1., one firm moves from low to normal, another two from normal to high and one from high to excellent; in group 3.2, two firms move from normal to high and one from high to excellent, in 4.1, one firm changes from poor to low and another from normal to high; in 4.3, one moves from low to normal; in group 5.1, two entities are transformed from high to excellent. In this component, 15 changes of firms occur, which improve the level of their human resources retention factor.

These results demonstrate that the impact of each component on the retention configuration may be understood and, in consequence, strategic decisions may be taken according to the objectives that are pursued. Thus, the question is how to influence those behaviours and actions, of which the firm may be unaware or may be ignoring, once they have been identified in the analysis. In concrete, there is a wide range of possibilities that runs from designing and applying selection policies that take into account the needs for knowledge within the firm, until the actions are proposed that allow the employees to become aware of, to understand, and to make the value of the organizational culture their own.

Likewise, the firm should be aware that the retention configuration calls for the provision of help so that the incorporation of new employees is a success, as well as for meetings and events to promote relations between veteran staff and recent arrivals. In this sense, integration policies should be based on the particularities of the people, knowledge exchange and sharing, the establishment of teams of people with different levels experience and service and the context in which the work will develop. Finally, as made clear, the policies of remuneration and motivation are of vital importance to achieve an excellent level of human resource retention.

A comparison of CMLHL projections with those obtained from some other unsupervised techniques: Principal Component Analysis (PCA) [39], MLHL and Self-Organizing Map (SOM) [41] were also applied to the HR dataset previously analyzed. Only those projections obtained by CMHL have been included in present study as they are more sparse [2] [35] and the data ordering can be identified in a more clear way. 


\section{Conclusions and Future Work}

The objective that prompted us to conduct this study was twofold: on the one hand, to prove the validity of the technique, for a set of qualitative, abstract and disordered data, representative of the level reached in the retention configuration of $R \& D$ personnel, in a significant sample of Spanish high-tech firms (126), and, on the other hand, to contribute a necessary element of rigor and robustness to research in the field of KM. Accordingly, it was possible to scale up from theoretical formulations to the application tools, the purpose of which is first of all to diagnose the reality and, then, to shed light on or to guide certain actions that improve their application or implementation in the firm.

This objective may be said to have been achieved because the application of the tool has served not only to group together and to order the data, the interpretation of which is highly significant, but also to arrive at interesting results, which serve to take decisions that can assure the effectiveness of the knowledge retention configuration. This HR practice has a high-level impact on competitiveness and the survival of the firm. From among these results, the following may be highlighted:

1. Clear identification of the firm's position with regard to its employee retention factor, which at the same time emerges from the configuration components attributed to it. This allows us to represent and, in consequence, to interpret the importance or commitment that the firms in this study attribute to this point, representative of a working base with the necessary knowledge and capabilities, whether economic, sustainable and stable, and in which the best professionals from each area are concentrated and maintained. Simultaneously, together with the preceding point on the position of the firm, the representation of the components or the variables of this factor provide information on the category or level in each configuration component and, therefore, help determine which achieve the best values and in which intervention is necessary. More specifically, the results allow us to establish, for example, if the firm has a plan where the needs of specific abilities are specified for the available posts and if higher salaries are offered to contract the best worker, where this salary is an element of retention. Likewise, it establishes if the selection is done on the basis of the global adjustment of the firm, if the employees identify with the organizational culture and whether there is a plan for the integration of new employees. All these elements work towards the success of human resource retention in the firm.

2. The convergence of various variables, each one with identical weightings, in clearly identified areas or spaces, which represent business positions or situations with regard to different levels of the retention factor; descending from excellent, high, and normal, to low and then poor. And their comparison, when the weighting is different for each of the five variables under analysis, maintaining the rest respectively equal. The displacement of the data set to other slightly different zones, as well as its variation in the primary score that was given, establish the characteristics of the firm, and those that it can manage to achieve, according to the actions that it undertakes, to situate itself, according to each case, in the best positions, changing from one to another, according to the employee retention strategy that is needed or relinquishing strategies that have proved inefficient. 
Corchado

In brief, the tool applied to the data used in this analysis has to a high degree of accuracy detected the position, the level and the components that configure working practice for the retention of $R \& D$ employees in a significative sample of Spanish firms, and the way in which it may evolve and progress towards a more effective introduction and execution, which will achieve higher levels of competitiveness and value. Acceptable behavior of the tool, in this and in earlier works, opens a wide field of possibilities for data treatment in fields where these are of a qualitative, abstract, and disordered nature, which will without doubt contribute to improving their diagnosis and may, more importantly, serve to advance applied research, providing guidance to business managers and executives.

Future work will focus on extending this study to some other factors that are important in the HR and KM fields (described in Section 2) and on the application of other unsupervised visualization models.

Acknowledgments. This research was partially supported through grants awarded by the Spanish Ministry of Economy and Competitiveness (ref: TIN2010-21272-C02-01) funded by the European Regional Development Fund, and by the Junta de Castilla y León (ref: SA405A12-2).

\section{References}

1. Herrero, Á., Corchado, E., Sáiz, L., Abraham, A.: DIPKIP: A Connectionist Knowledge Management System to Identify Knowledge Deficits in Practical Cases. Computational Intelligence 26(1), 26-56 (2010)

2. Durst, S., Edvardsson, I.R.: Knowledge Management in SMEs: a Literature Review. Journal of Knowledge Management 16(6), 879-903 (2012)

3. Pandey, S.C., Dutta, A.: Role of Knowledge Infrastructure Capabilities in Knowledge Management. Journal of Knowledge Management 17(3), 435-453 (2013)

4. Levy, M.: Knowledge Retention: Minimizing Organizational Business Loss. Journal of Knowledge Management 15(4), 582-600 (2011)

5. Becker, G.S.: Human Capital. Columbia University Press (1964)

6. Jiang, K., Lepak, D.P., Han, K., Hong, Y., Kim, A., Winkler, A.-L.: Clarifying the Construct of Human Resource Systems: Relating Human Resource Management to Employee Performance. Human Resource Management Review 22(2), 73-85 (2012)

7. Mowday, R.T.: Reflections on the Study and Relevance of Organizational Commitment. Human Resource Management Review 8(4), 387-401 (1999)

8. Kalchschmidt, M.: Best Practices in Demand Forecasting: Tests of Universalistic, Contingency and Configurational Theories. International Journal of Production Economics 140(2), 782-793 (2012)

9. Boon, C., Den Hartog, D.N., Boselie, P., Paauwe, J.: The Relationship between Perceptions of HR Practices and Employee Outcomes: Examining the Role of Person-organisation and Person-job Fit. The International Journal of Human Resource Management 22(01), 138-162 (2011)

10. Tremblay, M., Vandenberghe, C., Doucet, O.: Relationships Between Leader-Contingent and Non-contingent Reward and Punishment Behaviors and Subordinates' Perceptions of Justice and Satisfaction, and Evaluation of the Moderating Influence of Trust Propensity, Pay Level, and Role Ambiguity. Journal of Business and Psychology 28(2), 233-249 
11. Tremblay, M., Cloutier, J., Simard, G., Chênevert, D., Vandenberghe, C.: The Role of HRM Practices, Procedural Justice, Organizational Support and Trust in Organizational Commitment and In-role and Extra-role Performance. The International Journal of Human Resource Management 21(3), 405-433 (2010)

12. Aguinis, H., Joo, H., Gottfredson, R.K.: What Monetary Rewards Can and Cannot Do: How to Show Employees the Money. Business Horizons 56(2), 241-249 (2013)

13. Lam, W., Chen, Z., Takeuchi, N.: Perceived Human Resource Management Practices and Intention to Leave of Employees: the Mediating Role of Organizational Citizenship Behaviour in a Sino-Japanese Joint Venture. The International Journal of Human Resource Management 20(11), 2250-2270 (2009)

14. Gifford, R.H.: An Analysis of the Market Response to Announcements of Broad-based Stock Option Plans and an Analysis of the Effects of Broad-based Plans on Firm Performance, Employee Behavior and Employee Retention. University Microfilms International (2002)

15. Širca, N.T., Babnik, K., Breznik, K.: Towards Organisational Performance: Understanding Human Resource Management Climate. Industrial Management \& Data Systems 113(3), 367-384 (2013)

16. Haesli, A., Boxall, P.: When Knowledge Management Meets HR Strategy: an Exploration of Personalization-retention and Codification-recruitment Configurations. The International Journal of Human Resource Management 16(11), 1955-1975 (2005)

17. Ellinger, A.E., Musgrove, C.C.F., Ellinger, A.D., Bachrach, D.G., Elmadağ Baş, A.B., Wang, Y.-L.: Influences of Organizational Investments in Social Capital on Service Employee Commitment and Performance. Journal of Business Research (2012)

18. Mamman, A., Kamoche, K., Bakuwa, R.: Diversity, Organizational Commitment and Organizational Citizenship Behavior: An Organizing Framework. Human Resource Management Review 22(4), 285-302 (2012)

19. Zatzick, C.D., Iverson, R.D.: Putting Employee Involvement in Context: a Cross-level Model Examining Job Satisfaction and Absenteeism in High-involvement Work Systems. The International Journal of Human Resource Management 22(17), 3462-3476 (2011)

20. Wood, S., Van Veldhoven, M., Croon, M., de Menezes, L.M.: Enriched Job Design, High Involvement Management and Organizational Performance: The Mediating Roles of Job Satisfaction and Well-being. Human Relations 65(4), 419-445 (2012)

21. Herold, D.M., Fedor, D.B., Caldwell, S., Liu, Y.: The Effects of Transformational and Change Leadership on Employees' Commitment to a Change: A Multilevel Study. Journal of Applied Psychology 93(2), 346-356 (2008)

22. de Menezes, L.M., Wood, S., Gelade, G.: The Integration of Human Resource and Operation Management Practices and its Link with Performance: a Longitudinal Latent Class Study. Journal of Operations Management 28(6), 455-471 (2010)

23. Mowday, R.T., Steers, R.M., Porter, L.W.: The Measurement of Organizational Commitment. Journal of Vocational Behavior 14(2), 224-247 (1979)

24. Clark, K., Lengnick-Hall, M.L.: MNC Practice Transfer: Institutional Theory, Strategic Opportunities and Subsidiary HR Configuration. The International Journal of Human Resource Management 23(18), 3813-3837 (2012)

25. Kehoe, R.R., Wright, P.M.: The Impact of High-Performance Human Resource Practices on Employees' Attitudes and Behaviors. Journal of Management 39(2), 366-391 (2013)

26. Meyer, J.P., Hecht, T.D., Gill, H., Toplonytsky, L.: Person-organization (Culture) Fit and Employee Commitment under Conditions of Organizational Change: a Longitudinal Study. Journal of Vocational Behavior 76(3), 458-473 (2010)

27. Brown, M.M.: An Exploratory Study of Job Satisfaction and Work Motivation of a Select Group of Information Technology Consultants in the Delaware Valley. Wilmington College (2002) 
14 Lourdes Sáiz, Miguel Á. Manzanedo, Arturo Pérez, Álvaro Herrero and Emilio

Corchado

28. Reiche, B.S.: The Configuration of Employee Retention Practices in Multinational Corporations' Foreign Subsidiaries. International Business Review 17(6), 676-687 (2008)

29. Shaw, D., Edwards, J.S.: Building User Commitment to Implementing a Knowledge Management Strategy. Information \& Management 42(7), 977-988 (2005)

30. Parden, R.J.: The Manager's Role and the High Mobility of Technical Specialists in the Santa Clara Valley. IEEE Transactions on Engineering Management (1), 2-8 (1981)

31. McClean, E., Collins, C.J.: High-commitment HR Practices, Employee Effort, and Firm Performance: Investigating the Effects of HR Practices across Employee Groups within Professional Services Firms. Human Resource Management 50(3), 341-363 (2011)

32. Nishii, L.H., Lepak, D.P., Schneider, B.: Employee Attributions of the "why" of HR Practices: Their Effects on Employee Attitudes and Behaviors, and Customer Satisfaction. Personnel Psychology 61(3), 503-545 (2008)

33. Sáiz, L., Pérez, A.: Formación de las Capacidades de Creación de Conocimiento y Flexibilidad Organizativa en Empresas de Alta Tecnología. In: 4th International Conference on Industrial Engineering and Industrial Management. pp. 800-809 (2010)

34. Dolan, S.L., Mach, M., Olivera, V.S.: HR Contribution to a Firm's Success Examined from a Configurational Perspective: An Exploratory Study Based on the Spanish CRANET Data. Management Review 16(2), 272 (2005)

35. Herrero, Á., Corchado, E., Jiménez, A.: Unsupervised Neural Models for Country and Political Risk Analysis. Expert Systems with Applications In Press (2011)

36. Herrero, Á., Corchado, E., Gastaldo, P., Zunino, R.: Neural Projection Techniques for the Visual Inspection of Network Traffic. Neurocomputing 72(16-18), 3649-3658 (2009)

37. Friedman, J.H., Tukey, J.W.: A Projection Pursuit Algorithm for Exploratory DataAnalysis. IEEE Transactions on Computers 23(9), 881-890 (1974)

38. Hotelling, H.: Analysis of a Complex of Statistical Variables into Principal Components. Journal of Education Psychology 24, 417-444 (1933)

39. Pearson, K.: On Lines and Planes of Closest Fit to Systems of Points in Space. Philosophical Magazine 2(6), 559-572 (1901)

40. Seung, H.S., Socci, N.D., Lee, D.: The Rectified Gaussian Distribution. Advances in Neural Information Processing Systems 10, 350-356 (1998)

41. Kohonen, T.: The Self-Organizing Map. Proceedings of the IEEE 78(9), 1464-1480 (1990) 